\title{
Variación de la presión intraocular y medidas cuantitativas del segmento anterior pre y postiridotomía en pacientes sospechosos de cierre angular primario
}

\section{Intraocular pressure variation and quantitative assessment of the anterior chamber pre- and post-iridotomy in primary angle-closure suspects}

\author{
Tatiana Gil-Martínez ${ }^{1}$ María E. Brazón²*, Olga R. Cedeño y Cáceres Alfonso ${ }^{3}$ \\ ${ }^{1}$ Centro Diagnóstico Oftalmológico de Oriente; ${ }^{2}$ Centro de Oftalmología Dra. Mirna Hernández; ${ }^{3}$ Grupo de Investigación de Modelos Estadísticos \\ Aplicados a la Medicina (GIMEM), Universidad de Oriente (UDO). Anzoátegui, Venezuela
}

\section{Resumen}

Objetivos: Evaluar los cambios a corto plazo que acontecen después de una iridotomía láser periférica en la presión intraocular (PIO) y el ángulo camerular mediante tomografía de coherencia óptica de segmento anterior en pacientes sospechosos de cierre angular primario (PACS). Métodos: Estudio prospectivo, observacional, comparativo. Se incluyeron individuos PACS según la Clasificación de Foster. Los parámetros evaluados antes y después de la iridotomía fueron PIO, profundidad de cámara anterior, ángulo trabecular iris (TIA), distancia de apertura angular (AOD500) y área del espacio trabecular iris (TISA500) con tomografía de segmento anterior. Resultados: Se analizaron 28 ojos de 14 pacientes, se encontró una disminución de la PIO de $16.6 \pm 3.1$ a $14.4 \pm 4.3 \mathrm{mmHg}(p=0.001)$ pre y postiridotomía. También se encontró un incremento estadísticamente significativo $(p=0.001)$ en los valores de TIA, TISA500 y AOD500 $(p=0.032)$. Conclusiones: Este estudio confirma que, en pacientes con sospecha de cierre angular, la iridotomía láser periférica resulta en un incremento significativo de la amplitud del ángulo de la cámara anterior, así como una disminución significativa de la PIO.

Palabras claves: Sospechosos de cierre angular primario. Iridotomía láser periférica. Profundidad de cámara anterior. Tomografía de coherencia óptica del segmento anterior.

\section{Abstract}

Objectives: To evaluate the short-term changes that occur after laser peripheral iridotomy on intraocular pressure (IOP) and chamber angle by anterior segment optical coherence tomography in primary angle-closure suspects (PACS). Methods: Prospective, observational, comparative study. PACS were included according to the classification proposed by foster. The parameters evaluated before and after the iridotomy were IOP, anterior chamber depth, trabecular-iris angle (TIA), angle opening distance (AOD500) and trabecular-iris space area (TISA500) using anterior segment tomography. Results: We analyzed 28 eyes of 14 patients and found a decrease in IOP from $16.6 \pm 3.1$ to $14.4 \pm 4.3 \mathrm{mmHg}(p=0.001)$ pre- and post-iridotomy.

Correspondencia:

*María E. Brazón

Prolongación C. Arismendi. Centro Empresarial

Colón. 2 으. № $^{\circ}$

Fecha de recepción: 04-05-2018

Fecha de aceptación: 08-10-2018 DOI: 10.24875/RMO.M18000054
Disponible en internet: 02-01-2019 Rev Mex Oftalmol. 2019;93(1):14-18 www.rmo.com.mx cia CC BY-NC-ND 
We also found a statistically significant increase $(p=0.001)$ in TIA,TISA500 and AOD500 values $(p=0.032)$. Conclusion: This study confirms that, in PACS, peripheral laser iridotomy results in a significant increase in the anterior chamber angle amplitude, as well as a significant decrease in IOP.

Key words: Primary angle-closure suspect. Laser peripheral iridotomy. Anterior chamber depth. Anterior segment optical coherence tomography.

\section{Introducción}

El glaucoma primario por cierre angular (GPAC) se estima que es responsable de aproximadamente la mitad de la ceguera en todo el mundo'. Aproximadamente el $22 \%$ de los pacientes asiáticos con sospecha de cierre angular primario (PACS) evolucionaran a cierre angular primario (PAC) en 5 años y el $28 \%$ de estos desarrollarán GPAC ${ }^{2}$. El conocimiento de la configuración del ángulo de la cámara anterior es una parte esencial del diagnóstico y manejo de los pacientes glaucomatosos ${ }^{3}$. La gonioscopia es la técnica de referencia para su evaluación ${ }^{4,5}$, ya que permite la valoración de detalles como presencia de pigmento, depósitos, material pseudoexfoliativo, así como la diferenciación entre el cierre aposicional del sinequial; aunque presenta inconvenientes, como su subjetividad, lo que la hace muy dependiente del examinador, y la consiguiente baja reproducibilidad, además de la habilidad requerida por parte del evaluador y la adecuada colaboración por parte del paciente ${ }^{3,6}$.

Existen algunas alternativas para la evaluación del ángulo camerular, entre ellas la tomografía de coherencia óptica del segmento anterior (OCT-SA), que ha mostrado ser confortable y de gran resolución espacial a este nivel anatómico ${ }^{7-9}$.

La iridotomía láser periférica (ILP) es el procedimiento de elección en caso de PACS y PAC, dado que causa un aumento significativo en la apertura del ángulo de la cámara anterior ${ }^{10,11}$. El objetivo de este estudio es evaluar los cambios a corto plazo que ocurren después de una iridotomía en la presión intraocular (PIO) y en el ángulo camerular mediante OCT-SA en pacientes PACS.

\section{Metodología}

Estudio prospectivo, observacional, comparativo, en 31 ojos de 17 pacientes que acudieron a la consulta del Centro Diagnóstico Oftalmológico de Oriente y Unidad Oftalmológica Dra. Mirna Hernández desde mayo de 2016 hasta mayo de 2017. Previamente, los pacientes firmaron el consentimiento informado, se obtuvo la aprobación del comité de ética del colegio de médicos del estado Anzoátegui y se acataron los lineamientos de la declaración de Helsinki.

Se incluyeron pacientes mayores de 18 años y menores de 78, con ametropías esféricas de -3.00 a +6.00 dioptrías y cilindros inferiores a 3 dioptrías, con PIO normal < $21 \mathrm{mmHg}$ y diagnóstico de PACS según Clasificación de Foster ${ }^{12}$.

Fueron excluidos los pacientes con antecedentes de cirugía intraocular previa, traumatismo ocular, presencia de sinequias anteriores periféricas, cristalino intumescente, uveítis, que estuvieran recibiendo tratamiento médico hipotensor o que presentaran alteraciones sugestivas de neuropatía óptica glaucomatosa.

A todos los participantes se les realizó evaluación oftalmológica completa, incluyendo: agudeza visual (cartilla de Snellen), biomicroscopia, tonometría de aplanación de Goldmann, gonioscopia, fundoscopia (lente de 78D), paquimetría ultrasónica de contacto (marca Tomey modelo SP-100). Los parámetros biométricos, profundidad de cámara anterior (ACD) y longitud axial (LA), fueron tomados con biómetro de no contacto IOL master (Marca Carl Zeiss). Todos los exámenes los llevó a cabo el mismo oftalmólogo.

La evaluación gonioscópica se realizó con lente de 4 espejos de apoyo corneal, se instiló proparacaína tópica al $0.5 \%$, y se empleó baja iluminación ambiental. Con el paciente sentado en la lámpara de hendidura, en posición primaria de la mirada, el haz de luz de $1 \mathrm{~mm}$ se orientó horizontalmente para evaluar el ángulo nasal y temporal; mientras que para evaluar el superior e inferior, el haz de luz se dirigió verticalmente. Si la porción pigmentada de la malla trabecular no era visible en $270^{\circ}$, se consideraba un ojo con PACS según Foster ${ }^{12}$.

El equipo empleado para la adquisión de imágenes del segmento anterior fue el OCT RTVue ${ }^{\circledR}$ (Optovue Inc., Fremont, CA), y el lente empleado para medirlas fue el CAM-L. Se evaluaron los cuadrantes nasal y temporal (ángulo $0^{\circ}$ y $180^{\circ}$ ) en condiciones mesópicas. Se capturaron imágenes con el paciente sentado con la mirada hacia el lado contrario del ángulo a explorar. Se aceptaron solo las imágenes de calidad que indicaban intensidad de potencia de señal mayor a 30. Cada cuadrante se escaneó dos veces y se escogió la 
imagen que mostraba la mejor calidad, el menor ruido y donde se identificaran con más facilidad la mayor cantidad de estructuras anatómicas.

Las mediciones tomadas en consideración fueron: ángulo trabecular iris (TIA), distancia de apertura angular (AOD500) y el área del espacio trabecular iris (TISA500), estas dos últimas tomadas a 500 micras desde el espolón escleral.

EI TIA se definió mediante el trazado de una línea desde el receso del ángulo a la línea de Schwalbe y otra línea en la superficie del iris al punto perpendicular en la línea de Schwalbe. El AOD500 se determinó como la distancia perpendicular desde la malla trabecular, 500 micras en sentido anterior desde el espolón escleral a la superficie anterior del iris. EI TISA500 se definió como el área comprendida entre la AOD 500, la superficie del iris, el endotelio corneal y una línea perpendicular que parte desde el espolón escleral hasta el iris ${ }^{3,13}$.

Después de la toma de las medidas basales, se procedió a realizar la iridotomía. Para su realización se instiló pilocarpina al $2 \%$, pasados 30 minutos se colocó proparacaína tópica al $0.5 \%$, y se utilizó el lente de Abraham con gel de acoplamiento. Se seleccionó una cripta en el $1 / 3$ externo del iris periférico ubicada en el sector superior, de preferencia en el cuadrante nasal, hasta observar salida de humor acuoso y pigmento. Para el procedimiento se empleó láser YAG (itrium-aluminio-granate) modelo LIGHTLas YAG de LIGHTMED Corporation. El poder fue ajustado entre 3 a $6 \mathrm{~mJ}$. Se reevaluó la PIO 1 hora después al procedimiento, se indicó prednisolona tópica 1\%, 1 gota cada 8 horas durante 6 días y control en 1 semana. La PIO, parámetros biométricos y OCT-SA fueron reevaluados 1 a 4 semanas después de la iridotomía.

\section{Análisis estadístico}

Se calculó la media aritmética y desviación típica de las variables continuas: edad, esfera, cilindro, paquimetría, LA, PIO, ACD, TIA, TISA500 y AOD500. AI comparar los promedios se aplicó la prueba de $t$ de Student de datos apareados en edad por género, TIA, TISA500 y AOD500, en los grupos pre y postiridotomía. La prueba de Shapiro-Wilk determinó el comportamiento paramétrico $(p>0.05)$ de estas variables. En la comparación de las medias de las variables, PIO y $A C D$, entre los grupos pre y postiridotomía, se aplicó la prueba de Wilcoxon por presentar los datos tendencia no paramétrica de acuerdo a la prueba de Shapiro-Wilk $(p<0.05)$. Se computó frecuencia y porcentaje de la variable categórica, género del paciente. Los
Tabla 1. Medidas de la presión intraocular y profundidad de cámara anterior pre y postiridotomia

\begin{tabular}{|l|c|c|c|}
\hline Variable & Preiridotomía & Postiridotomía & \multicolumn{1}{c|}{ P } \\
\hline PIO (mmHg) & $16.6 \pm 3.1$ & $14.4 \pm 4.3$ & 0.001 \\
\hline ACD $(\mathrm{mm})$ & $2.7 \pm 0.3$ & $2.8 \pm 0.03$ & 0.401 \\
\hline
\end{tabular}

Los valores son media aritmética más/menos desviación típica. ACD: profundidad de cámara anterior; PIO: presión intraocular.

datos se analizaron con el paquete estadístico computarizado SPSS versión 21.0. La validez estadística se ubicó en un nivel de $p<0.05$.

\section{Resultados}

Al final se analizaron 28 ojos, los 2 ojos de 14 pacientes, con el fin de homogeneizar adecuadamente la muestra, se excluyeron 3 pacientes que tenían solo un ojo en condiciones de análisis. La edad media fue de $56.4 \pm 11.1$ años, con un rango de edad entre $39 \mathrm{y}$ 78 años, no hubo diferencia estadística entre hombres y mujeres $(p=0.106)$. De la muestra, 9 pacientes (64.3\%) fueron mujeres. En cuanto al error refractivo, la media de la esfera fue de $1.84 \pm 1.8$ dioptrías, con un rango de -1 a 6 , y la del cilindro de $-0.49 \pm 0.50$ dioptrías, con un rango -1.5 a 0 .

De los 28 ojos evaluados, la media del espesor corneal central fue de $543.2 \pm 45.9 \mu \mathrm{m}$, con un rango de 416 a $620 \mu \mathrm{m}$; la media de la longitud axial fue de $22.7 \pm 0.65 \mathrm{~mm}$, con un rango de 21.5 a 24.1 .

La variación de la PIO fue de $16.6 \pm 3.1$ a $14.4 \pm 4.3 \mathrm{mmHg}(p=0.001)$ pre y postiridotomía, respectivamente, lo que representa una disminución estadísticamente significativa. El promedio de la ACD antes y 4 semanas después de la iridotomía, incrementó de $2.7 \pm 0.3$ a $2.8 \pm 0.2 \mathrm{~mm}(p=0.401)$. Este último dato no fue estadísticamente significativo (Tabla 1).

En la tabla 2 se presentan los valores de TIA, TISA500 y AOD500, medidos tanto en el ángulo nasal como temporal, antes y después al tratamiento con iridotomía. Los valores de TIA, TISA500 y AOD500 mostraron un incremento estadísticamente significativo $(p=0.001)$, excepto el TISA500 en el ángulo nasal, que no muestra relevancia estadística $(p=0.032)$.

\section{Discusión}

El objetivo de este estudio fue evaluar los cambios a corto plazo que acontecen después de iridotomía en la PIO y en el ángulo camerular mediante OCT-SA en 
Tabla 2. Cambios en los parámetros angulares antes y después de la iridotomía láser periférica

\begin{tabular}{|c|c|c|c|c|c|c|}
\hline \multirow[t]{2}{*}{ Variable } & \multicolumn{2}{|c|}{ Ángulo nasal } & \multirow[t]{2}{*}{$\mathbf{p}$} & \multicolumn{2}{|c|}{ Ángulo temporal } & \multirow[t]{2}{*}{$\mathbf{p}$} \\
\hline & Preiridotomía & Postiridotomía & & Preiridotomía & Postiridotomía & \\
\hline $\operatorname{TIA}\left({ }^{\circ}\right)$ & $12.0 \pm 5.1$ & $18.2 \pm 6.9$ & 0.001 & $11.2 \pm 6.3$ & $18.2 \pm 6.4$ & 0.001 \\
\hline TISA500 $\left(\mathrm{mm}^{2}\right)$ & $0.107 \pm 0.19$ & $0.117 \pm 0.20$ & 0.032 & $0.055 \pm 0.03$ & $0.086 \pm 0.04$ & 0.001 \\
\hline A0D500 $(\mu \mathrm{m})$ & $136.8 \pm 81.0$ & $208.0 \pm 103.3$ & 0.001 & $143.9 \pm 80.1$ & $221.3 \pm 105.4$ & 0.001 \\
\hline
\end{tabular}

Los valores son media aritmética más/menos desviación típica.

AOD: distancia de apertura angular; TIA: ángulo trabecular iris; TISA: área espacio trabecular iris.

pacientes PACS. Además, se examinó el efecto de la iridotomía en la $A C D$, utilizando un biómetro de no contacto. La OCT-SA es una técnica de excelente resolución, de no contacto y de gran facilidad de uso. A pesar de la amplia aceptación de la gonioscopia como el patrón oro en la evaluación del ángulo camerular, las mediciones hechas por OCT-SA tienen como ventaja la realización de análisis cuantitativos, poder hacer medidas en córneas opacas, en posición sentada y en pacientes poco colaboradores, además de ser altamente reproducible ${ }^{14-16}$.

Los resultados obtenidos en este estudio muestran que después de la iridotomía, hubo un aumento significativo del ángulo camerular, que quedó evidenciado por un aumento del TIA, TISA500 y AOD500, similar a lo reportado por diversos autores quienes han publicado sus resultados tras estudiar diversas razas de pacientes y empleando para ello diversos equipos de imagen para medición de parámetros del ángulo de la cámara anterior ${ }^{17-19}$.

How, et al. ${ }^{19}$ evaluaron 176 ojos confirmando un aumento de la AOD y TISA posterior a ILP medida por OCT Visante. Ang et al., por su parte, evaluaron 71 ojos de pacientes caucásicos y reportó una ampliación significativa del ángulo camerular periférico medido con OCT-SA Slit lamp ${ }^{17}$. Lee, et al..$^{20}$ obtuvieron similares resultados en 32 ojos de pacientes PACS. Este estudio confirma lo publicado por anteriores investigaciones $^{17-21}$, que el ángulo camerular se ensancha claramente en ojos de PACS después de la iridotomía.

En este estudio, la medición del TISA nasal no presentó ningún cambio significativo, similar a lo reportado por Jiang, et al. ${ }^{22}$ quienes encontraron que el $25 \%$ de los ojos tenían persistencia del ángulo cerrado después de la iridotomía, posiblemente las razones de estos hallazgos pueden ser explicadas por la existencia de otros factores que afectan la apertura angular distintos al grado de bloqueo pupilar, como son la posición o grosor del iris y el cuerpo ciliar, el cristalino, entre otros, cuya medición escapaban a los objetivos planteados al inicio de este estudio ${ }^{22}$.

Existe la controversia sobre el efecto de la iridotomía en la $A C D^{21,23-25}$. Este estudio demostró que, en ojos de PACS, la iridotomía no tiene efecto significativo en la ACD, al igual que fue mostrado por Faramarzi, et al. quienes evaluaron 40 ojos de PACS mediante biómetro Lemstar $^{26}$.

Un estudio con Pentacam de 20 ojos de PACS mostró una diferencia significativa en el volumen de la cámara anterior, TIA y ACD periférica, pero no en la ACD central, después de realizarse iridotomía ${ }^{23}$.

Con respecto al efecto de la iridotomía sobre la presión intraocular, en este estudio, la presión disminuyó significativamente después del procedimiento, a diferencia de lo reportado por Vryonis et al. ${ }^{27}$, quienes estudiaron 46 ojos de PACS, PAC y GPAC y no demostraron un descenso de la PIO estadísticamente significativa. Probablemente, esta diferencia se deba a que en su muestra incluyeron pacientes con PAC y GPAC, mientras que para este estudio solo se incluyeron pacientes PACS, los cuales por definición presentan niveles de presión intraocular normal.

Haciendo referencia a las limitaciones encontradas en el presente trabajo, tenemos, en primer lugar, el tamaño de la muestra, que nos impide generalizar los resultados obtenidos; en segundo lugar, la dificultad en la identificación del espolón escleral en algunos ojos, bien por ser anatómicamente poco evidente, o por razones de reflectividad o contraste, y en tercer lugar, el no haber considerado otros parámetros como el volumen del lente y grosor iridiano en las medidas empleadas, que nos hubiesen permitido entender mejor las razones por las cuales en algunos pacientes el procedimiento no se obtuvo el efecto deseado de apertura angular.

Con los resultados obtenidos se puede concluir que la iridotomía produce un incremento significativo en el ángulo en ojos de PACS y una disminución significativa de la PIO. 


\section{Responsabilidades éticas}

Protección de personas y animales. Los autores declaran que los procedimientos seguidos se conformaron a las normas éticas del comité de experimentación humana responsable y de acuerdo con la Asociación Médica Mundial y la Declaración de Helsinki.

Confidencialidad de los datos. Los autores declaran que han seguido los protocolos de su centro de trabajo sobre la publicación de datos de pacientes.

Derecho a la privacidad y consentimiento informado. Los autores han obtenido el consentimiento informado de los pacientes y/o sujetos referidos en el artículo. Este documento obra en poder del autor de correspondencia.

\section{Conflicto de intereses}

Los autores declaran que no tienen conflicto de intereses con ninguna de las casas comerciales de los instrumentos y equipos empleados para el estudio.

\section{Bibliografía}

1. Quigley HA, Broman AT. The number of people with glaucoma worldwide in 2010 and 2020. Br J Ophthalmol. 2006;90(3):262-7.

2. Pérez H, García Y, Gómez N. Cierre angular primario: ópciones quirúrgicas. Rev Mex Oftalmol. 2014;88(4):182-5.

3. Radhakrishnan S, Goldsmith J, Huang D, Westphal V, Dueker DK, Rollins AM, Izatt JA. Comparision of optical coherence tomography and ultrasound biomicroscopy for detection of narrow anterior chamber angle. Arch Ophthalmol. 2005;123:1053-9

4. Xu L, Cao WF, Wang YX y cols. Anterior chamber depth and chamber angle and their associations with ocular and general parameters: The Beijing Eye Study. Am J Ophthalmol 2008;145(5):929-936.

5. Sakata LM, Lavanya R, Friedman DS, Aung HT, Gao H, Kumar RS, et al. Comparison of gonioscopy and anterior segment ocular coherence tomography in detecting angle closure in different quadrants of the anterior chamber angle. Ophthalmology. 2008;115:769-74.

6. Kalev-Landoy M, Day AC, Cordeiro MF Migdal C. Optical coherence tomography in anterior segment imaging. Acta Ophthalmol Scand. 2007; 85(4):427-30.

7. Leung CK, Chan WM, Ko CY, Chui SI, Woo J, Tsang MK, et al. Visualization of anterior chamber angle dynamics using optical coherence tomography. Ophthalmology. 2005;112:980-4.

8. Dada T, Sihota R, Gadia R, Aggarwal A, Mandal S, Gupta V. Comparison of anterior segment optical coherence tomography and ultrasound biomicroscopy for assessment of the anterior segment. J Cataract Refract Surg. 2007;33:837-40

9. Mansouri K, Sommerhalder J, Shaarawy T. Prospective comparison of ultrasound biomicroscopy and anterior segment optical coherence tomography for evaluation of anterior chamber dimensions in European eyes with primary angle closure. Eye (Lond). 2010;24:233-9.
10. Hsiao $\mathrm{CH}$, Hsu CT, Shen SC, Chen HS. Mid-term follow-up of Nd: YAG laser iridotomy in Asian eyes. Ophthalmic Surg Lasers Imaging. 2003; 34(4):291-8.

11. Jiang $Y$, Chang DS, Zhu H, Khawaja AP, Aung T, Huang S, et al. Longitudinal Changes of Angle Configuration in Primary Angle-Closure Suspects: The Zhongshan Angle-Closure Prevention Trial. Ophthalmology. 2014;121(9):1699-705.

12. Foster PJ, Buhrmann R, Quigley HA, Johnson GJ. The definition and classification of glaucoma in prevalence surveys. $\mathrm{Br} \mathrm{J}$ Ophthalmol. 2002;86:238-42.

13. Sun JH, Sung KR, Yun SC, Cheon MH, Tchah HW, Kim MJ, et al. Factors associated with anterior chamber narrowing with age: an optical coherence tomography study. Invest Ophthalmol Vis Sci. 2012;53:2607-10.

14. Li H, Leung CK, Cheung CY, Wong L, Pang CP, Weinreb RN, et at. Repeatability and reproducibility of anterior chamber angle measurement with anterior segment optical coherence tomography. Br J Ophthalmol. 2007;91:1490-2.

15. Muller M, Dahmen G, Porksen E, Geerling G, Laqua H, Ziegler A, et al. Anterior chamber angle measurement with optical coherence tomography: intraobserver and interobserver variability. J Cataract Refract Surg. 2006;32:1803-8.

16. Radhakrishnan S, See J, Smith SD, Nolan WP, Ce Z, Friedman DS, et al Reproducibility of anterior chamber angle measurement obtained with anterior segment optical coherence tomography. Invest Ophthalmol Vis Sci. 2007;48:3683-8.

17. Ang GS, Wells AP. Changes in Caucasian eyes after laser peripheral iridotomy: an anterior segment optical coherence tomography study. Clin Exp Ophthalmol. 2010;38:778-85

18. Gazzard G, Friedman DS, Devereux JG, Chew P, Seah SK. A prospective ultrasound biomicroscopy evaluation of changes in anterior segment morphology after laser iridotomy in Asian eyes. O phthalmology 2003; 110:630-8.

19. How A, Baskaran M, Kumar R, He M, Foster P, Lavanya R, et al. Changes in Anterior segment morphology after laser peripheral Iridotomy: An anterior segment optical coherence tomography study. Ophthalmology. 2012;119(7):1383-7.

20. Lee KS, Sung KR, Shon K, Sun JH, Lee JR. Longitudinal Changes in anterior segment parameters after peripheral iridotomy assessed by anterior segmnet optical coherence tomography. Invest Ophthal Vis Sci. 2013;54(5):3166-70.

21. Lopez-Caballero C, Puerto-Hernández B, Muñoz-Negrete FJ, Rebolleda G, Contreras I, Cabarga C, Corral A. Quantitative evaluation of anterior chamber changes after iridotomy using Pentacam anterior segment analyser. Eur J Ophthalmol. 2010;20:327-32.

22. Jiang $\mathrm{Y}$, Chang DS, Zhu H, Khawaja AP, Aung T, Huang S, et al. Longitudinal Changes of angle configuration in Primary Aanle Closure Suspects: The Zhongshan Angle-Closure Prevention Trial. Ophthalmology.2014;121(9):1699-705.

23. Antoniazzi E, Pezzotta S, Delfino A, Bianchi PE. Anterior chamber measurements taken with Pentacam: an objetive tool in laser iridotomy. Eur J Ophthalmol. 2010;20:517-22.

24. Lis $\mathrm{S}$, Wang $\mathrm{H}, \mathrm{Mu} \mathrm{D}, \mathrm{Fu} \mathrm{J}$, Wang $\mathrm{X}$, Wang N. Prospective evaluation of changes in anterior segment morphology after laser iriditomy in Chinese eyes by rotationg Scheimplug camera imaging. Clin Experiment Ophtalmol. 2010;38:10-4.

25. Lei K, Wang N, Wang L, Wang B. Morphological changes of the anterior segment after laser peripheral iridotomy in primary angle closure. Eye (Lond). 2009;23:345-50.

26. Faramarzi A, Yazdani S, Pakravan M. Central anterior chamber Depth changes after prophylactic laser iridotomy. Optometry and Vision Science. 2013;90(7):707-10.

27. Vryonis N, Nikita E, Vergados I, Thoedossiadis P, Filippopoulos T. Anterior chamber morphology before and after laser peripheral iridotomy determined by Schimpfflug technology in white patients with narrow angles. J Glaucoma. 2013;22(9):679-83. 